\title{
DISCRIMINAÇÃO DE DOSES DE NITROGÊNIO EM MUDAS DE TECA USANDO SISTEMA DE SENSORIAMENTO REMOTO DE BAIXO CUSTO
}

\author{
Carolina de Faria Cabral Paes Pereira e Barros ${ }^{1}$, Selma Alves Abrahão ${ }^{2}$, Jacqueline \\ Miranda Ferreira ${ }^{1}$, Ronann de Oliveira Gomes de Arruda ${ }^{1}$, Wagner da Cunha \\ Siqueira ${ }^{2}$ \\ ${ }^{1}$ Graduando(a) do Curso Bacharelado em Engenharia Florestal do Instituto Federal \\ de Mato Grosso - Campus Cáceres (carolinna.ef@gmail.com) Cáceres - MT, Brasil. \\ ${ }^{2}$ Professor(a) Doutor(a) do Curso Bacharelado em Engenharia Florestal do Instituto \\ Federal de Mato Grosso - Campus Cáceres, Cáceres - MT, Brasil.
}

\section{Recebido em: 08/09/2015 - Aprovado em: 14/11/2015 - Publicado em: 01/12/2015 \\ DOI: http://dx.doi.org/10.18677/Enciclopedia_Biosfera_2015_271}

\begin{abstract}
RESUMO
Objetivou-se com esse trabalho discriminar doses de nitrogênio $(\mathrm{N})$ de mudas de teca utilizando bandas espectrais obtidas por um sistema de sensoriamento remoto de baixo custo, definindo as melhores épocas e variáveis. Buscou-se também estudar o efeito das doses de $\mathrm{N}$ sobre o teor de $\mathrm{N}$ foliar e peso de massa seca de raízes e da parte aérea de mudas de teca. Foram avaliadas quatro doses de $\mathrm{N}$ aplicadas em cobertura $\left(0,50,100\right.$ e $\left.200 \mathrm{mg} \mathrm{dm}^{-3}\right)$, em um delineamento inteiramente casualizado, com 10 repetições (exceto para o tratamento $200 \mathrm{mg} \mathrm{dm}^{-3}$ de $\mathrm{N}$, com 9 repetições). O sistema de sensoriamento remoto foi constituído por uma câmera digital de pequeno formato, utilizada para adquirir imagens do dossel das plantas nas bandas do vermelho $(R)$, verde $(G)$ e azul $(B)$, em seis épocas, 21,28 , 35, 42, 48 e 56 dias após o plantio (56 DAP). Para discriminar as doses de $\mathrm{N}$ foram testados classificadores do tipo função discriminante quadrática. Os classificadores demonstraram serem eficientes na discriminação das doses de $\mathrm{N}$ aplicadas em cobertura. As melhores épocas encontradas para se obterem imagens e discriminar as doses de $\mathrm{N}$ foram aos 48 e 56 DAP, com o Kappa variando de 0,2424 a 0,3853. Aos 62 DAP, as mudas de teca demonstraram respostas significativas à aplicação crescente das doses de $\mathrm{N}$ para teor de $\mathrm{N}$ foliar e peso de massa seca parte aérea, exceto para o peso de massa seca de raiz.
\end{abstract}

PALAVRAS-CHAVE: adubação nitrogenada, classificadores, processamento de imagens

\section{DISCRIMINATION OF NITROGEN RATES IN THE TEAK SEEDLINGS USING LOW-COST REMOTE SENSING SYSTEM}

\begin{abstract}
The objective of this study was discriminating nitrogen $(\mathrm{N})$ rates in the teak seedlings using spectral bands, using low-cost remote sensing system, and to define the best time and best variables. The objective of this study was to evaluate the effect of nitrogen rates on leaf nitrogen, dry mass of shoot and dry mass of roots in the teak
\end{abstract}


seedlings. Four nitrogen levels $\left(0,50,100\right.$ and $\left.200 \mathrm{mg} \mathrm{dm}^{-3}\right)$ were evaluated in a completely randomized design with 10 replications (except for treating $200 \mathrm{mg} \mathrm{dm}^{-3}$ of $\mathrm{N}$, with 9 replications). The remote sensing system consisted of a small-format digital camera, used to acquire the plant canopy images in the bands of red $(R)$, green (G) and blue (B) in six times, 21, 28, 35 , 42, 48 and 56 days after planting (56 DAP). In this work, quadratic discriminant analysis was accomplished for classification of nitrogen $(\mathrm{N})$ rates in the teak seedlings. The classifiers proved to be efficient in determining nitrogen rates. The best times for determining nitrogen rates were at 48 DAP and 56 DAP, with a Kappa ranging from 0.2424 to 0.3853 . The nitrogen rates significantly affected the on leaf nitrogen and dry mass of shoot in the teak seedlings 62 DAP, except for the dry mass of roots.

KEYWORDS: nitrogen fertilization, classifiers, image processing

\section{INTRODUÇÃO}

A teca (Tectona grandis L. f.) é uma das espécies mais utilizadas em reflorestamentos no estado de Mato Grosso, pois apresenta um elevado valor comercial da sua madeira (SILVA et al., 2013), resistência às pragas e doenças e taxas de crescimento superiores às dos plantios da maioria dos países produtores dessa madeira, devido as condições ambientais adequadas (PASSOS et al., 2006). $O$ interesse no cultivo da teca pode também ser demonstrado pelo aumento da área plantada no estado do Mato Grosso, estimada em mais de 50 mil hectares (HACKBARTH et al., 2014).

Segundo KANNUR \& DEVAR (2004), mudas de qualidade são imprescindíveis para o sucesso do plantio de teca, principalmente em solos pobres. FREIBERGER et al. (2013) estudaram o crescimento inicial e nutrição em mudas de cedro em função de doses de $\mathrm{N}$ aos 210 DAP, em condição de vaso. Segundo os autores, os incrementos em altura, diâmetro de colo e peso de massa seca das mudas de cedro em razão do aumento das doses de $\mathrm{N}$ evidenciam a importância da adubação nitrogenada no desenvolvimento inicial dessa espécie. BARROSO et al. (2005) estudando as deficiências de macronutrientes em mudas de teca aos 30 dias após o plantio observaram que os danos mais intensos ocorreram com a deficiência de nitrogênio $(\mathrm{N})$ e cálcio, sendo que no tratamento com omissão de $\mathrm{N}$ houve redução drástica do crescimento, clorose generalizada, paralisação de emissão de raízes novas e apodrecimento das raízes secundárias.

Para conseguir maior eficiência no uso do $\mathrm{N}$ e de outros nutrientes na produção, torna-se necessário conhecer a época mais apropriada e de maior exigência pelas plantas, a dose adequada de aplicação desse nutriente e onde aplicar. Técnicas de Silvicultura de Precisão têm sido propostas com a finalidade de otimizar o uso de nutrientes para diversas espécies florestais.

O Sensoriamento Remoto tem se mostrado como uma das técnicas mais promissoras para a Silvicultura de Precisão. Ao contrário do método tradicional, permite adquirir informações dos objetos de forma não destrutiva, sem que haja contato físico direto com o mesmo, em larga escala e em tempo real (ABRAHÃO et al., 2013).

Diversos trabalhos têm sido realizados com o desenvolvimento e avaliação de dados espectrais (bandas e índices de vegetação) para determinação das relações destes com as características biofísicas do dossel das espécies florestais, tais como volume de madeira (FERNANDES et al., 2011; BERRA et al., 2012), altura, diâmetro à altura do peito (FERNANDES et al. 2011), índice de área foliar e temperatura 
(WANDERLEY et al., 2013), todos em espécies de eucalipto. Entretanto, estudos sobre teca ainda são escassos, principalmente no Estado de Mato Grosso, em que se demonstra alta perspectiva de retorno financeiro.

Objetivou-se com esse trabalho discriminar as doses de $\mathrm{N}$ de mudas de teca utilizando bandas espectrais obtidas por um sistema de sensoriamento remoto de baixo custo, definindo as melhores épocas e variáveis. Objetivou-se também estudar o efeito das doses de $\mathrm{N}$ sobre o teor de $\mathrm{N}$ foliar e peso de massa seca de raízes e da parte aérea de mudas de teca.

\section{MATERIAL E MÉTODOS}

O experimento foi conduzido em casa de vegetação pertencente ao Instituto Federal de Educação, Ciência e Tecnologia de Mato Grosso - Campus Cáceres, Cáceres, MT, durante os meses de janeiro e março de 2014. Foram utilizadas mudas de Tectona grandis L.f. (teca) do tipo clone, com, aproximadamente, 3 pares de folhas e altura média de $15 \mathrm{~cm}$, fornecidas pela empresa EMPAER, situada na cidade de Cáceres.

As mudas foram transplantadas para 40 vasos ( $2 \mathrm{dm}^{3}$ de solo por vaso) em $15 / 01 / 2014$, contendo uma muda por vaso. O solo utilizado apresentou as seguintes características: $72,48,12,63$ e $14,89 \mathrm{~g} \mathrm{~kg}^{-1}$ de areia, silte e argila, respectivamente; $\mathrm{pH}=5,7 ; \mathrm{P}=2,81 \mathrm{mg} \mathrm{dm}^{-3} ; \mathrm{K}=19,71 \mathrm{mg} \mathrm{dm}^{-3} ; \mathrm{Ca}=3,1 \mathrm{cmol}_{\mathrm{c}} \mathrm{dm}^{-3} ; \mathrm{Mg}=0,70$ $\mathrm{cmol}_{\mathrm{c}} \mathrm{dm}^{-3} ; \mathrm{Al}=0,0 \mathrm{cmol}_{\mathrm{c}} \mathrm{dm}^{-3}$; soma de bases $=3,9 \mathrm{cmol}_{\mathrm{c}} \mathrm{dm}^{-3}$; capacidade de troca de cátions a pH 7,0 $=6,0 \mathrm{cmol}_{\mathrm{c}} \mathrm{dm}^{-3}$; saturação por bases $=64,7 \%$; matéria orgânica $=5,9 \mathrm{~g} / \mathrm{kg}$.

Considerando os resultados da análise do solo, realizou-se adubação uniforme em todos os vasos: $75,04 \mathrm{~g}$ de $\mathrm{Ca}\left(\mathrm{H}_{2} \mathrm{PO}_{4}\right)_{2}, 15 \mathrm{~g}$ de $\mathrm{KCl}, 0,082 \mathrm{~g}$ de $\mathrm{H}_{3} \mathrm{BO}_{3}, 0,133 \mathrm{~g}$ de $\mathrm{CuSO}_{4} \cdot 5 \mathrm{H}_{2} \mathrm{O}, 0,154 \mathrm{~g}$ de $\mathrm{FeSO}_{4} .7 \mathrm{H}_{2} \mathrm{O}, 0,013 \mathrm{~g}$ de $\mathrm{H}_{2} \mathrm{MoO}_{4}, 0,23$ $\mathrm{g}$ de $\mathrm{MnCl}_{2} .4 \mathrm{H}_{2} \mathrm{O}, 0,40 \mathrm{~g}$ de $\mathrm{ZnSO}_{4} .7 \mathrm{H}_{2} \mathrm{O}$ e $5 \mathrm{~g}$ de $\mathrm{C}_{6} \mathrm{H}_{8} \mathrm{O}_{7}$ dissolvidos em $5 \mathrm{dm}^{3}$ de água e aplicados 7 dias após o plantio das mudas.

Foi utilizado um delineamento inteiramente casualizado, com quatro tratamentos e dez repetições (exceto para o tratamento $200 \mathrm{mg} \mathrm{dm}^{-3}$ de nitrogênio, com 9 repetições). Os tratamentos foram compostos pelas quatro doses de Nitrogênio (N) aplicadas em cobertura $\left(0,50,100\right.$ e $\left.200 \mathrm{mg} \mathrm{dm}^{-3}\right)$, na forma de ureia $(44 \%$ de $N)$, aos 7 dias após o plantio das mudas.

A irrigação do experimento foi realizada diariamente, de forma a manter a umidade do solo a $80 \%$ do ponto de saturação.

$\mathrm{O}$ sistema de sensoriamento remoto foi utilizado para adquirir imagens do dossel das mudas de teca, nas bandas do vermelho $(R)$, verde $(G)$ e azul $(B)$, em seis épocas, 21 dias após o plantio (21 DAP), 28 DAP, 35 DAP, 42 DAP, 48 DAP e 56 DAP. Foi utilizada uma câmera digital da Sony, modelo cyber-shot DSC-W180, 10,1 megapixels, equipada com um único CCD de 1/2,3". As configurações das câmeras foram: formato JPEG; dimensões de 3648 por 2736 pixels; modo de disparo manual; flash, antiborramento, detecção de face inteligente e remoção dos olhos vermelhos desligados; compensação de exposição $\pm 0 \mathrm{EV}$; sensibilidade ISO 800; balanço de branco automático; modo foco automático centro.

As imagens foram adquiridas em condições de iluminação controlada. Para tanto, foi utilizada uma estrutura metálica, com largura e profundidade de $1,0 \mathrm{~m}$ e altura de 1,2 m, recoberta com tecido do tipo "blackout" e um sistema de iluminação composto por quatro refletores com lâmpadas halógenas de 50 watts. Os refletores foram direcionados para cima para evitar incidência de luz direta sobre as mudas. 
Os vasos foram posicionados individualmente no interior da estrutura para obtenção das imagens.

As imagens foram segmentadas pelo método de limiarização Otsu (OTSU, 1974) para retirar o efeito do fundo. As imagens antes de serem segmentadas, foram processadas com o índice de excesso de verde normalizado $(E v d)$, adaptado de MEYER et al. (1998):

$$
E v d=(4 \times G-R-B) /(G+R+B)
$$

Para discriminar as classes (definidas pelas doses de $\mathrm{N}$ aplicadas em cobertura na adubação) foram testados classificadores multivariados do tipo função discriminante quadrática. Os classificadores foram desenvolvidos para cada época de aquisição de imagens, para cada banda ( $R$ e $G$ ) e para as combinações das bandas, definindo a melhor época e as melhores variáveis para discriminar as classes.

A função discriminante é derivada do teorema de Bayes e estima a probabilidade a posteriori, $P\left(w_{j} \mid X\right)$, de que um vetor $X$ seja classificado na classe $w_{j}$ (JOHNSON; WICHERN, 1998). Essa probabilidade é obtida para cada uma das classes $w_{j}$, por meio do produto da probabilidade a priori de ocorrência da classe, $P\left(w_{j}\right)$, pela probabilidade condicional dada as características $X$ dentre da classe $w_{j}$, $P\left(X \mid w_{j}\right)$. O individuo é classificado na classe que apresentar maior valor de $P\left(w_{j} \mid X\right)$.

Dessa maneira, a probabilidade $P\left(w_{j} \mid X\right)$, considerando uma covariância para cada classe, é obtida pela seguinte função discriminante quadrática:

$D_{j}(X)=-\frac{1}{2}\left(X-\bar{X}_{j}\right)^{T}\left(\hat{\Sigma}_{j}\right)^{-1}\left(X-\bar{X}_{j}\right)-\frac{1}{2} \ln \left|\hat{\Sigma}_{j}\right|+\ln \left[P\left(w_{j}\right)\right]$

em que,

$D_{j}(X)=$ valor da função discriminante quadrática da classe $w_{j}$ dado $X$;

$X \quad=$ vetor de característica de um indivíduo;

$\bar{X}_{j} \quad=$ vetor médio da classe $j$;

$\widehat{\Sigma}_{j} \quad=$ estimativa da matriz de covariância comum da classe $j$;

$\left|\varepsilon_{j}\right|=$ determinante da estimativa da matriz de covariância comum da classe $j$;

$P\left(w_{j}\right)=$ probabilidade a priori de ocorrência da classe $j$.

A probabilidade a priori $P\left(w_{j}\right)$ foi estimada pela frequência de ocorrência das classes na amostra.

Os resultados das classificações foram avaliados elaborando-se a matriz de erros, utilizando a técnica de validação cruzada (deixando uma amostra de fora), e calculando-se o coeficiente Kappa (HUDSON \& RAMM, 1987). Os classificadores foram avaliados pelo teste $Z$ dos seus coeficientes Kappa, com nível de significância de 5\%. Para auxiliar na análise dos classificadores, os mesmos também foram classificados em: Ruim quando o valor da estimativa do coeficiente Kappa está entre 0,00 e 0,19; Razoável entre 0,20 e 0,39; Bom entre 0,40 e 0,59; Muito bom entre 0,60 e 0,79; e Excelente quando o valor de Kappa é maior ou igual a 0,80 (LANDIS $\& \mathrm{KOCH}, 1977)$. 
Aos 62 DAP, em cada vaso foram coletadas, aleatoriamente, três folhas no terço médio da muda de teca, para determinar o teor de $\mathrm{N}$ foliar em laboratório pelo método de Kjeldahl. Também aos 62 DAP todas as mudas foram cortadas à altura do solo e separadas em raízes e parte aérea. As mudas foram lavadas, pesadas e postas para secar em estufa de ventilação forçada à $65^{\circ} \mathrm{C}$, até atingirem peso constante. Em seguida, foram determinados: peso da massa seca de raízes (PMSR) e da parte aérea (PMSPA), utilizando uma balança de precisão 0,0001 mg.

Com o intuito de conhecer o efeito das doses de $\mathrm{N}$ sobre teor de $\mathrm{N}$ foliar, PMSR e PMSPA foram realizadas análises de variância das regressões com o teste F para falta de ajustamento para cada época de avaliação, com nível de significância de $5 \%$. Não foram testados modelos de regressão lineares superiores ao segundo grau, por estes não representarem o fenômeno biológico da cultura, pois em geral, a resposta com a elevação de doses de N é quadrática.

\section{RESULTADOS E DISCUSSÃO}

Aos 21, 28, 35 e 42 DAP, nenhum classificador apresentou coeficiente Kappa estatisticamente diferente de zero pelo teste $Z$ ao nível de $5 \%$ de probabilidade. $O$ resultado não satisfatório pode estar relacionado com o estádio de desenvolvimento das mudas de teca, no início, apesar das unidades experimentais terem sido submetidas a diferentes doses de $\mathrm{N}$ na adubação, estas diferenças não tiveram efeito na resposta espectral das bandas.

Aos 48 DAP, dos três classificadores testados, somente um apresentou coeficiente Kappa estatisticamente diferente de zero pelo teste $Z$ ao nível de $5 \%$ de probabilidade. O classificador selecionado utilizou como variável de entrada a banda $R$ (kappa $=0,2414)$. De acordo com NAUE et al. (2010), as clorofilas a e b são os pigmentos que mais influenciam a radiação eletromagnética na região do visível, com dois picos de absorção, o maior na banda $R$ e o menor na banda $B$, respectivamente, favorecendo um melhor resultado para o classificador que utilizou a banda $\mathrm{R}$.

Aos 56 DAP, todos classificadores apresentaram coeficiente Kappa estatisticamente diferente de zero pelo teste $Z$ ao nível de $5 \%$ de probabilidade.

A qualidade das classificações foi considerada razoável (LANDIS \& $\mathrm{KOCH}$, 1977). A qualidade razoável pode estar relacionada com o estádio inicial de desenvolvimento das mudas de teca, pois o aumento de absorção de $\mathrm{N}$ nos estádios iniciais é lento, sendo, portanto, pouco armazenado e translocado (TA \& WEILAND, 1992). Isto indica que apesar das unidades experimentais terem sido submetidas a diferentes doses de $\mathrm{N}$, estas diferenças tiveram pouco efeito na resposta espectral das bandas.

O resultado razoável também pode estar relacionado às bandas serem mais aptas a discriminar doses de $\mathrm{N}$ onde está deficiente, fato não observado na análise de teor de $\mathrm{N}$ foliar realizada em laboratório aos 62 DAP (Tabela 2), as parcelas que receberam as doses 50,100 e $200 \mathrm{mg} \mathrm{dm}^{-3}$ de $\mathrm{N}$ apresentaram valores médios de teores de $\mathrm{N}$ foliar dentro da faixa de teores adequados para mudas de teca, indicando que as mudas de teca estavam bem nutridas. De acordo com FERNÁNDEZ-MOYA et al. (2013), a faixa de teores adequados preestabelecido para plantas de teca com idade de 5 anos, para os países Costa Rica e Panamá, esta entre 19,7 e $23,3 \mathrm{~g} \mathrm{~kg}^{-1}$.

Resultados semelhantes foram encontrados por ABRAHÃO et al. (2013) que desenvolveram classificadores com base em diferentes combinações de bandas e índices de vegetação espectrais de imagens originais, segmentadas e reflectâncias, 
para discriminação de teores de $\mathrm{N}$ e clorofila foliares do feijoeiro aos 13 dias após a emergência. Segundo os autores, estes dados são melhores para discriminar N onde estes estão deficientes, fato não observado no campo, todas as parcelas apresentaram teor de $\mathrm{N}$ foliar aos 13 DAE acima do nível crítico, entre 3,0 e 3,5 dag $\mathrm{kg}^{-1}$.

TABELA 1 Classificadores selecionados com base na estimativa do coeficiente Kappa

\begin{tabular}{cccc}
\hline Época & $\begin{array}{c}\text { Combinações } \\
\text { (variáveis de entrada) }\end{array}$ & Kappa & Classificação \\
\hline 48 DAP & $\mathrm{R}$ & $0,2414^{*} \mathrm{a}$ & Razoável \\
\hline \multirow{2}{*}{56 DAP } & $\mathrm{R}$ & $0,2771^{* *} \mathrm{a}$ & Razoável \\
\cline { 2 - 4 } & $\mathrm{G}$ & $0,2500^{* *} \mathrm{a}$ & Razoável \\
\cline { 2 - 4 } & $\mathrm{R} \mathrm{e} \mathrm{G}$ & $0,3853^{* *} \mathrm{a}$ & Razoável \\
\hline
\end{tabular}

** $\mathrm{e}^{*}$ : significativo ao nível de $1 \%$ e $5 \%$ de probabilidade, respectivamente, pelo teste Z; Os coeficientes kappa seguidos de uma mesma letra não diferiram pelo teste $Z$ ao nível de $5 \%$ de probabilidade.

TABELA 2 Valores médios de teor de nitrogênio $(N)$ foliar das mudas de teca em função das doses de $\mathrm{N}$ aplicadas no plantio aos $62 \mathrm{DAP}$

\begin{tabular}{cc}
\hline Doses de $\mathbf{N}\left(\mathbf{~ m g ~ d m}^{-3}\right)$ & Valores médios de teor de $\mathbf{N}$ foliar $\left.\mathbf{( g ~} \mathbf{~ g ~}^{-1}\right)$ \\
\hline 0 & 16,0 \\
\hline 50 & 19,0 \\
\hline 100 & 21,8 \\
\hline 200 & 25,0 \\
\hline
\end{tabular}

Como os quatro classificadores foram estatisticamente iguais pelo teste $Z$ ao nível de significância de $5 \%$ e três apresentaram o modelo mais simples e menor tempo computacional (ou seja, uma variável), esses três seriam recomendados para classificar doses de $\mathrm{N}$ aos 48 e 56 DAP. Deste modo, as melhores épocas encontradas para se obter imagens e discriminar as doses de $\mathrm{N}$ foram aos 48 e 56 DAP.

As mudas de teca demonstraram respostas significativas à aplicação crescente das doses de $\mathrm{N}$ para as variáveis: teor de $\mathrm{N}$ foliar e PMSPA, exceto para o PMSR, aos 62 DAP (Tabela 3). FAVARE (2010) estudou o efeito das doses de N aplicadas em cobertura (testemunha absoluta, 0, 40, 80, 120 e $160 \mathrm{mg} \mathrm{dm}^{-3}$ ) no peso de massa seca de mudas de teca (folhas, caule, raízes e total), aos 240 DAP, sob condição de vaso. O autor não encontrou efeito significativo das doses de $\mathrm{N}$ para o peso de massa seca de raízes, demonstrando que, no estádio de muda, o $\mathrm{N}$ não é o elemento mais importante à formação de raízes para esta espécie.

BARROSO et al. (2005) avaliaram o crescimento e o teor de nutrientes na parte aérea e de raízes de mudas de teca submetidas à solução nutritiva completa e às soluções com omissão alternada de $\mathrm{N}, \mathrm{P}, \mathrm{K}, \mathrm{Ca}, \mathrm{Mg}$ e $\mathrm{S}$, aos $30 \mathrm{DAP}$. Os autores verificaram que o PMSR não foi afetado pela omissão de macronutrientes, apesar dos sintomas visuais de deficiência mineral (no tratamento com omissão de $\mathrm{N}$ houve redução drástica do crescimento, clorose generalizada, paralisação de emissão de raízes novas e apodrecimento das raízes secundárias).

Para teor de $\mathrm{N}$ foliar houve efeito linear de $1^{\circ} \mathrm{grau}$, com maior acúmulo na maior dose aplicada de $\mathrm{N}\left(200 \mathrm{mg} \mathrm{dm}^{-3}\right)$ e para o PMSPA o efeito foi linear de $2^{\circ}$ grau, com o maior acúmulo na dose estimada de $110 \mathrm{mg} \mathrm{dm}^{-3}$ de $\mathrm{N}$. Estes resultados corroboraram com FAVARE (2010), que obteve o maior acúmulo de teor 
de $\mathrm{N}$ foliar na maior dose aplicada de $\mathrm{N}\left(160 \mathrm{mg} \mathrm{dm}^{-3}\right)$ e maior acúmulo de massa seca para folhas e caule nas doses estimadas 109 e $99 \mathrm{mg} \mathrm{dm}^{-3}$ de $\mathrm{N}$, respectivamente.

O PMSPA apresentou uma tendência de decréscimo com aplicação da dose situada acima de $110 \mathrm{mg} \mathrm{dm}^{-3}$, que pode estar relacionado a uma possível desequilíbrio nutricional da muda com altas doses de $\mathrm{N}$. O efeito negativo do excesso de $\mathrm{N}$ pode provocar diminuição na quantidade do nutriente recuperado pela muda de teca, em decorrência da maior desnitrificação do nutriente, bem como a indução de toxidez na muda provocada pelo excesso de $\mathrm{N}$.

FREIBERGER et al. (2013) estudaram o crescimento inicial e nutrição em mudas de cedro em função de doses de $\mathrm{N}\left(0,40,80,120\right.$ e $\left.160 \mathrm{mg} \mathrm{dm}^{-3}\right)$ aos 210 DAP, em condição de vaso, e verificaram que altura, diâmetro de colo e peso de massa seca (folha, caule, raiz e total) ajustaram-se a um modelo linear de $1^{\circ}$ grau crescente. Segundo os autores, os incrementos em altura, diâmetro de colo e peso de massa seca das mudas de cedro em razão do aumento das doses de $\mathrm{N}$ evidenciam a importância da adubação nitrogenada no desenvolvimento inicial dessa espécie.

De maneira geral, essas respostas devem-se ao fato de que grandes quantidades de $\mathrm{N}$ são requeridas pelas plantas, principalmente na fase inicial de desenvolvimento (MALAVOLTA et al., 1997).

TABELA 3 Resultados da análise de regressão para o peso de massa seca de raízes (PMSR) e da parte aérea (PMSPA) e para o teor de $\mathrm{N}$ foliar das mudas de teca em função das doses de $\mathrm{N}$ aplicadas no plantio aos 62 dias após o plantio

\begin{tabular}{ccccc}
\hline Variável & \multicolumn{2}{c}{ Teste F } & \multicolumn{2}{c}{ Teste t } \\
& Falta de ajuste & Regressão & $\mathbf{N}$ & $\mathbf{N}^{2}$ \\
\hline Teor de N foliar (ML1) & $0,3819^{\mathrm{ns}}$ & $26,7796^{\star *}$ & $5,1749^{\star *}$ & --- \\
PMSR (ML1 / ML2) & $0,0161^{\mathrm{ns}} / 0,0080^{\mathrm{ns}}$ & $0,6039^{\mathrm{ns}} / 0,3140^{\mathrm{ns}}$ & --- & -- \\
PMSPA (ML2) & $0,1925^{\mathrm{ns}}$ & $4,2781^{*}$ & $2,7082^{*}$ & $-2,2788^{*}$ \\
\hline
\end{tabular}

ML1: modelo linear de $1^{\circ}$ grau; ML2: modelo linear de $2^{\circ}$ grau; $\mathrm{N}$ : doses de $\mathrm{N}\left(\mathrm{mg} \mathrm{dm}^{-3}\right)$; ${ }^{\text {ns. }}$ : não significativo ao nível de $5 \%$ de probabilidade; ${ }^{*}{ }^{* *}$ : significativo ao nível de 1 e $5 \%$ de probabilidade, respectivamente.

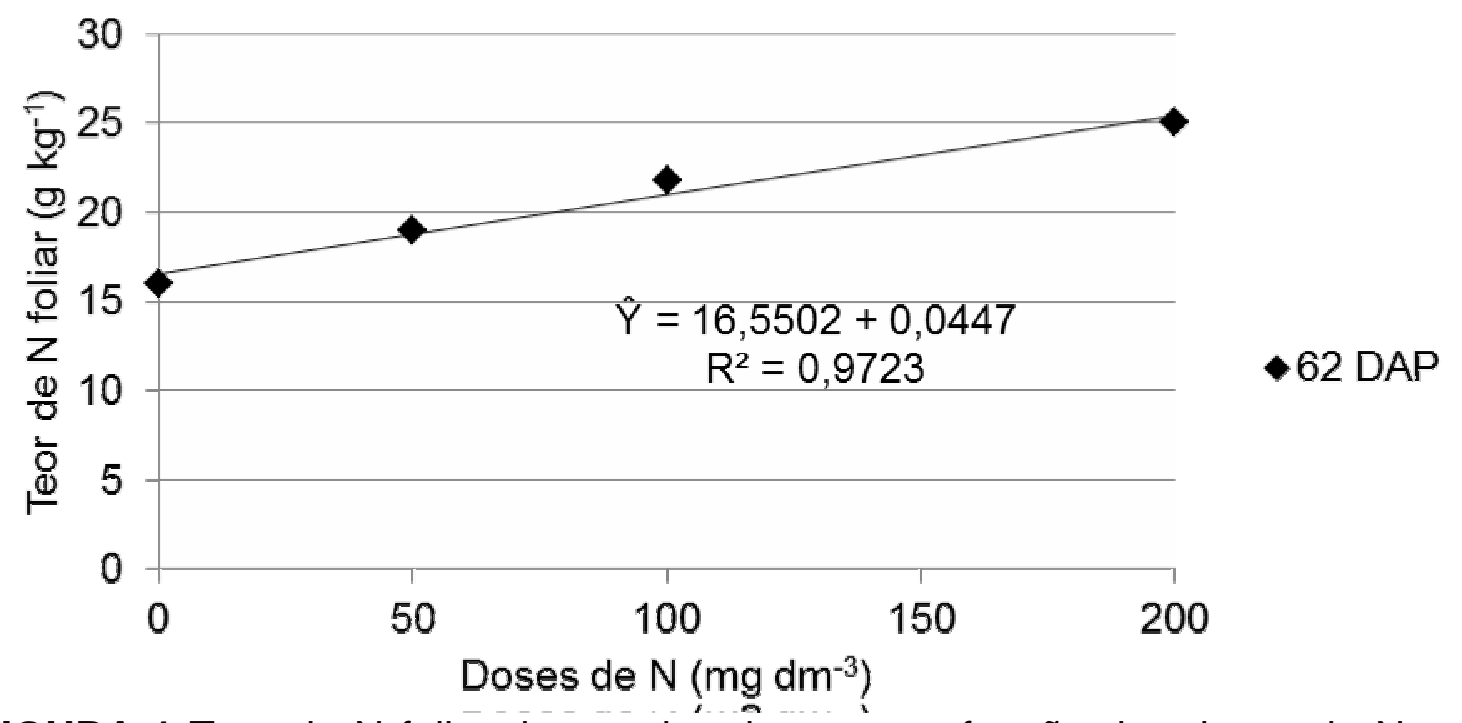

FIGURA 1 Teor de $\mathrm{N}$ foliar das mudas de teca em função das doses de $\mathrm{N}$ aos 62 dias após o plantio 


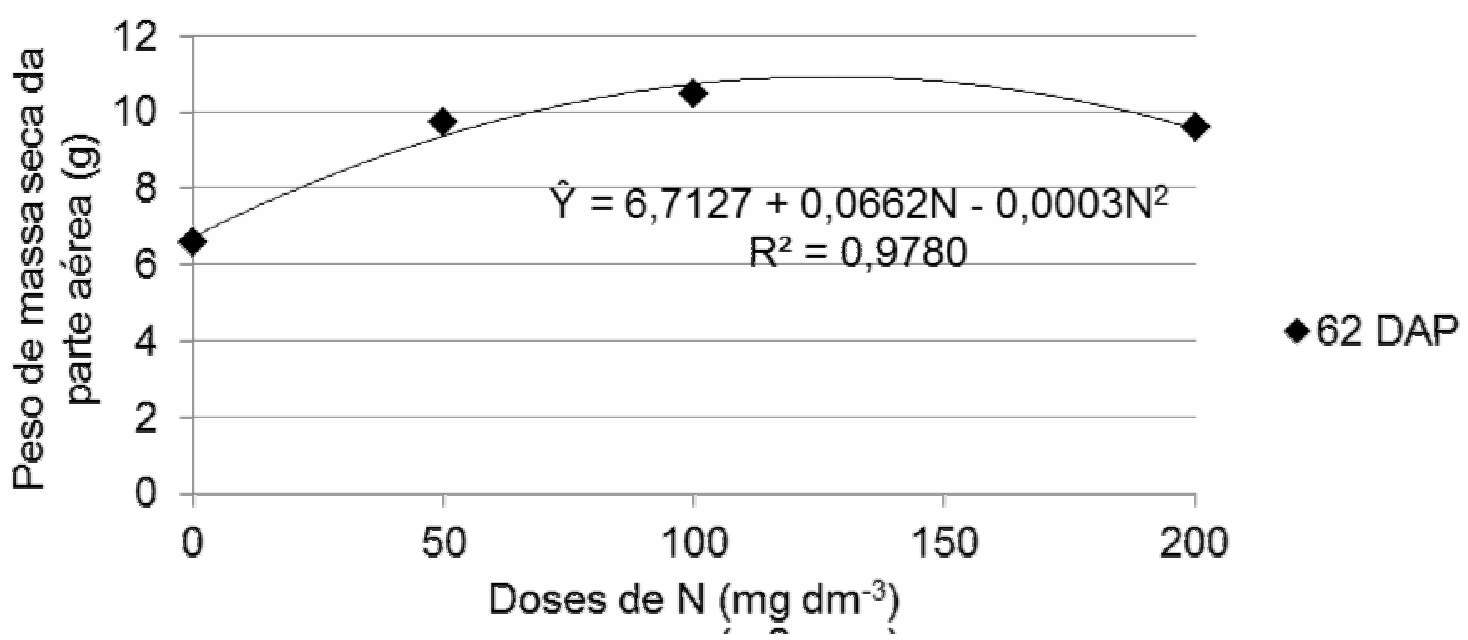

FIGURA 2 Peso de massa seca da parte aérea das mudas de teca em função das doses de $\mathrm{N}$ aos 62 dias após o plantio.

\section{CONCLUSÕES}

Os classificadores demonstraram serem eficientes na discriminação das doses de $\mathrm{N}$ aplicadas em cobertura em mudas de teca.

As melhores épocas encontradas para se obterem imagens e discriminar as doses de $\mathrm{N}$ foram aos 48 e 56 DAP, com o Kappa variando de 0,2424 a 0,3853.

As mudas de teca demonstraram respostas significativas à aplicação crescente das doses de $\mathrm{N}$ para as variáveis: teor de $\mathrm{N}$ foliar e peso de massa seca parte aérea, exceto para o peso de massa seca de raiz, aos 62 DAP.

\section{AGRADECIMENTOS}

Ao IFMT e à FAPEMAT, pela bolsa de pesquisa e o recurso financeiro.

\section{REFERÊNCIAS}

ABRAHÃO, S. A.; PINTO, F. A. C.; QUEIROZ, D. M.; SANTOS, N. T.; CARNEIRO, J. E. S. Determination of nitrogen and chlorophyll levels in bean-plant leaves by using spectral vegetation bands and indices. Revista Ciência Agronômica, v. 44, n. 3, p. 464-473, 2013.

BARROSO, D. G.; FIGUEIREDO, F. A. M. M. A.; PEREIRA, R. C.; MENDONÇA, A. V. R.; SILVA, L. C. Diagnóstico de deficiência de macronutrientes em mudas de Teca. Revista Árvore, v.29, n.5, p.671-679, 2005.

BERRA, E. F.; BRANDELERO, C.; PEREIRA, R. S.; SEBEM, E.; GOERGEN, L. C. G.; BENEDETTI, A. C. P.; LIPPERT, D. B. Estimativa do volume total de madeira em espécies de eucalipto a partir de imagens de satélite Landsat. Ciência Florestal, v.22, n.4, p. 853-864, 2012.

FAVARE, L. G. de. Doses crescentes de nitrogênio, fósforo, potássio e diferentes níveis de saturação por bases em relação ao desenvolvimento e 
nutrição mineral de teca (Tectona grandis L.f.), sob condições de vaso. 2010. 97f. Dissertação (Mestrado em Ciência Florestal) - Faculdade de Ciências Agronômicas da UNESP, Botucatu, São Paulo, 2010.

FERNANDES, D.H.F.; SOARES, V.P.; DE OLIVEIRA, F.S.; RIBEIRO, C.A.A.S.; GLERIANI, J.M. Uso de imagens ALOS para estimar parâmetros biofísicos em plantações de eucaliptos. In: Simpósio Brasileiro de Sensoriamento Remoto, 15, 2011, Curitiba, PR, Anais. Curitiba, INPE, 2011, p.1674.

FERNÁNDEZ-MOYA, J.; ALVARADO, A.; FORSYTHE, W.; MARCHAMALOSACRISTÁN, M. Effect of teak (Tectona grandis) plantations on hydraulic conductivity and porosity of alfisols in costa rica. Journal of Tropical Forest Science. Vol.25, n. 2, p. 259-267, 2013.

FREIBERGER, M. B.; GUERRINI, I. A.; GALETTI, G.; FERNANDES, D. M.; CORRÊA, J. C. Crescimento inicial e nutrição de Cedro (Cedrela fissilis Vell.) em função de doses de nitrogênio. Revista Árvore, v.37, n.3, p.385-392, 2013.

HACKBARTH, A.C.; BONALDO, S.M.; TRENTO, R.A.; RIBEIRO, A.S. Influência da concentração, do fotoperíodo e da temperatura de armazenamento na germinação de urediniósporos de Olivea tectonae. Biosci. J., v. 30, n. 2, p. 340-348, 2014.

HUDSON, W.D.; RAMM, C.W. Correct formulation of the Kappa coefficient of agreement. Photogrammetric Engineering \& Remote Sensing, v.53, n.4, 1987. JOHNSON, R. A.; WICHERN, D. W.; Applied Multivariate statistical analysis, 4. ed., Rio de Janeiro: Prentice-Hall, p. 816, 1999.

KANNUR, S.; DEVAR, K. V. Effect of Nitrogen on the Growth Performance of Teak Seedlings Nursery. Karnataka Journal of Agricultural Sciences, v.17, n.2, p. 358360, 2004.

LANDIS, J.R.; KOCH, G.G. The measurement of observer agreement for categorical data. Biometrics, v.33, n.1, 1977.

NAUE, C. R.; MARQUES, M. W.; LIMA, N. B.; GALVÍNCIO, J. D. Sensoriamento remoto como ferramenta aos estudos de doenças de plantas agrícolas: uma revisão. Revista Brasileira de Geografia Física, v.3, n.3, p. 190-195, 2010.

MALAVOLTA, E.; VITTI, G. C.; OLIVEIRA, S. A. Avaliação do estado nutricional das plantas, princípios e aplicações. 2.ed. Piracicaba: POTAFOS, p. 319, 1997.

MEYER, G.E.; MEHTA, T.; KOCHER, M.F.; MORTENSEN, D.A.; SAMAL, A. Textural imaging and discriminant analysis for distinguishing weeds for spot spraying. Transactions of the ASAE, v.41, n.4, 1998.

OTSU, N. A threshold selection method from gray-level histogram. IEEE Transactions on Systems, Man, and Cybernetics, p. 62-66, 1974. 
PASSOS, C. A. M.; BUFULIN JUNIOR, L.; GONÇALVES, M. R. Avaliação silvicultura de Tectona grandis L.f., Cáceres - MT, Brasil: Resultados preliminares. Ciência Florestal, v.16, n.2, p. 225-232, 2006.

SILVA, K. S.; RIBEIRO, E. S.; SOUZA, R. S.; CHAVES, A. G. S.; WEBER, O. L. S. Alterações das propriedades químicas de um solo reflorestado com Tectona grandis L.f. na região de nova Maringá, MT. Revista Eletrônica Geoaraguaia, Edição Especial, p.163-178, 2013.

TA, C. T.; WEILAND, R. T. Nitrogen partitioning in maize during ear development. Crop Science, v.32, n.2, p. 443-451, 1992.

WANDERLEY, R.L.N.; DA ROCHA, H.R.; CABRAL, O.M.R.; DE FREITAS, H.C.; BRASILIO, E.M.S.; DA SILVA, J.M. Estimativa do índice de área foliar e temperatura em uma área de eucalipto. Revista Ciência e Natura, Edição Especial, p. 386-389, 2013. 